\title{
Local Recurrence of Primary Cardiac Leiomyosarcoma After Resection: A Rare Case
}

\author{
Fatih Tay *, Mustafa Büyükkör, Öztürk Ateş \\ Health Science University, Dr. Abdurrahman Yurtaslan Ankara Oncology Training and Research Hospital, Ankara, Turkey
}

\section{ARTICLE INFO}

Received : 20 February 2021

Reviewed : 22 April 2021

Accepted : 21 March 2021

Keywords:

cardiac leiomyosarcoma, cardiac

tumors, soft tissue sarcomas

*Corresponding author:

Fatih Tay

Health Science University,

Dr. Abdurrahman Yurtaslan Ankara

Oncology Training and Research

Hospital, Ankara, Turkey

drfatihtay@gmail.com

\begin{abstract}
Introduction: Leiomyosarcoma (LMS) is a fatal tumor, though primary cardiac is very rarely encountered. In this report, we evaluated the surgical and medical management of primary cardiac LMS, which is a sporadic tumor.

Case Presentation: A 60-year-old female patient was examined for pulmonary edema after penicillin allergy in May 2019, while transthoracic echocardiography (ECHO) revealed an appearance compatible with a $5 \times 3.5 \mathrm{~cm}$ mass in the right atrium. In addition, transesophageal ECHO showed a $7 \times 4.6 \mathrm{~cm}$ foreground mass compatible with a myxoma in the right atrium (RA). The patient was then operated on emergency. Besides, an adjuvant ifosfamide-mesna-doxorubicine (IMA) protocol was planned to be given to the patient after local radiotherapy.
\end{abstract}

Conclusions: In this report presented, we made RT and chemotherapy treatment plans in the case of recurrence without RO surgery. Recurrence rates of up to $50 \%$ can occur in LMS, and systemic chemotherapy can be applied after relapse.

\section{INTRODUCTION}

Sarcomas constitute a rare and heterogeneous group of malignant tumors of mesenchymal origin, which make up less than $1 \%$ of all adult malignancies. About $80 \%$ of sarcoma cases originate from soft tissues, while the rest come from the bone. In this regard, the World Health Organization classifies most soft tissue neoplasms according to the tissue of origin. Examples include liposarcoma, synovial sarcoma, leiomyosarcoma (LMS), rhabdomyosarcoma (RMS), fibrosarcoma, and angiosarcoma. LMS is a fatal tumor, though primary cardiac is very rarely encountered [1]. Meanwhile, surgery is the best primary treatment in LMS, and the recurrence rate is not less. Systemic treatment regimens may also be used considering the presence of distant metastases.

In fact, the possibility of having a higher incidence may be neglected due to the lack of sufficient studies on primary cardiac sarcomas and the fact that most of the publications have been made as case reports. Therefore, our study aims to ensure that clinicians have more information about their treatment experiences, which will further increase the recommendations of any study on this subject. This view reveals the importance of our work.

\section{CASE PRESENTATION}

A 60-year-old female patient was examined for pulmonary edema after penicillin allergy in May 2019, while transthoracic echocardiography $(\mathrm{ECHO})$ revealed an appearance compatible with a $5 \times 3.5 \mathrm{~cm}$ mass in the right atrium. In addition, transesophageal ECHO showed a $7 \times 4.6 \mathrm{~cm}$ foreground mass compatible with a myxoma in the right atrium (RA). The patient was then operated on emergency. The tumor, which was evaluated as Leiomyosarcoma (LMS) grade 2, Ki-67 30\% by pathological examination, was $7 \times 5 \mathrm{~cm}$. Examination for systemic metastasis was performed by Positron Emission Tomography (PET-CT), and no distant metastasis was detected. The patient was followed up without medication. In the follow-up period, ECHO, taken approximately 17 months later, showed an appearance compatible with a $25 \times 22 \mathrm{~mm}$ mass, causing stenosis and insufficiency in the tricuspid valve entering and exiting the right atrium. Hypodense lesions of $41 \times 43$ $\mathrm{mm}$ in the right atrium and $55 \times 29 \mathrm{~mm}$ in the right ventricle were detected in CT angiography (Figure 1). On PET CT, heterogeneous increased FDG uptake in PET images at the level of the tricuspid valve in the right atrium adjacent to the operation area and a mass 
lesion suggesting local recurrence in the foreground were identified. The patient was then operated on for recurrence as she was symptomatic (Figure 2). In the operation note, it was stated that sternotomy had been performed to reach the right atrium, and the mass had extended into the inferior vena cava. The mass had been excised from the right atrium (Figure 3: excised mass). However, the lesion extending towards the inferior vena cava could not be removed entirely. CK was positive, Desmin was positive, Caldesmon was positive, Calretinin was weakly positive, and SMA (actin) focal was positive in the immunohistochemical examination following recurrence operation. Then, an adjuvant ifosfamide-mesna-doxorubicine (IMA) protocol was planned to be administered to the patient after local radiotherapy.

In this report presented, we made radiotherapy and chemotherapy treatment plans in the case of recurrence without R0 surgery. Recurrence rates of up to $50 \%$ can occur in LMS. Systemic chemotherapy can be applied after relapse.

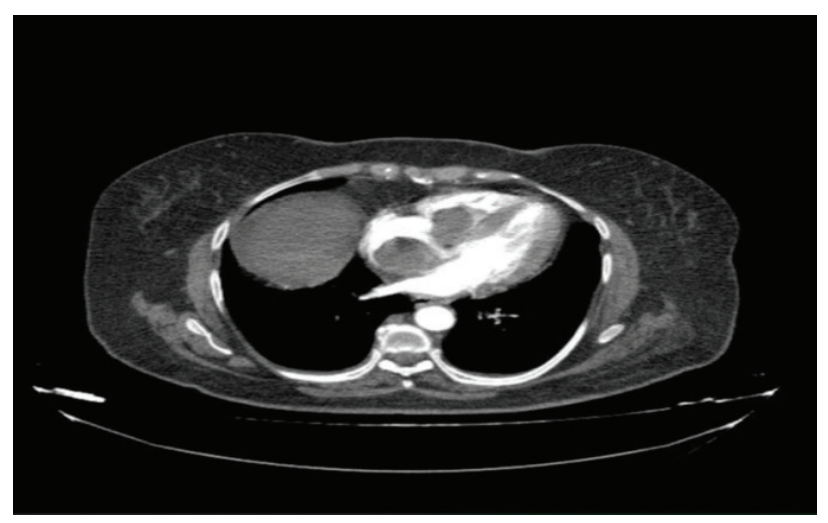

Figure 1. The hypodense lesion in the right ventricle

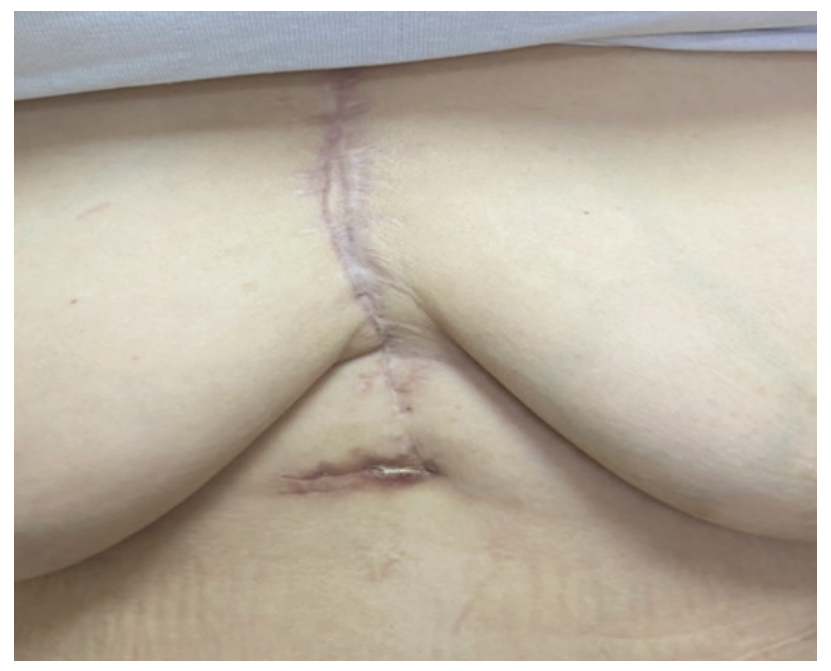

Figure 2. Post-recurrence sternotomy scar.

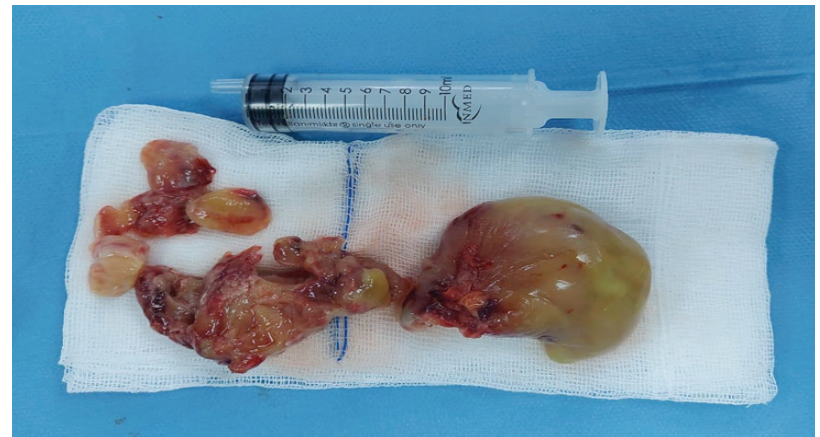

Figure 3. Macroscopic view of the excised mass.

\section{DISCUSSION}

A primary cardiac LMS case recurring 17 months after resection is presented. Although complete resection is the preferred treatment in cardiac sarcomas, most patients develop recurrence even after complete resection [2]. The risk of recurrence is much higher in incomplete resections. Even though the patient's clinical condition improves dramatically after incomplete resection, it is necessary to provide systemic treatment. As a systemic treatment, we planned to give our patient radiotherapy and subsequent chemotherapy.

In addition, primary cardiac LMS is very rarely encountered. It is observed in less than $0.25 \%$ of cardiac tumors $[3,4]$. Primary cardiac LMS usually presents with heart failure or embolism clinical signs. In our patient, the diagnosis was made during the examination due to dyspnea after an allergic reaction.

As in cases with benign lesions, the clinical status is mainly determined by the tumor location rather than its histopathology. The diagnostic approach relies on echocardiography, magnetic resonance (MR) imaging, and computed tomography (CT) to identify the presence of a tumor and its anatomical relationship with normal structures. However, current imaging is insufficient to distinguish between myxomas and sarcomas.

Moreover, the prognosis for cardiac LMS is generally poor, and the survival of primary cardiac LMS without treatment is approximately 6-12 months [5]. LMS is a spindle cell, a high-grade tumor more frequently occurring in the left atrium [6]. These sarcomas have both high local recurrence and systemic spread. Adriamycin, epirubicin, and ifosfamide are the primary drugs associated with more than $20 \%$ response rates for chemotherapy of metastatic soft tissue sarcoma. Several different combination chemotherapies, including these essential drugs, have been studied and shown to have a higher treatment response and more prolonged progression-free survival (PFS) than single regimens [7].

Then, we planned to give radiotherapy and a subsequent adjuvant IMA treatment to our patient after 
the second recurrence surgery. Unfortunately, our patient had developed local recurrence 17 months after the first diagnosis. Our patient is symptom-free and is still being followed up after local recurrence surgery and radiotherapy.

In this regard, there are publications that the average survival with the disease is 6-12 months [8], but our patient completed 17 months without symptoms after surgery, despite having a high-grade tumor. After her second surgery, the patient is still alive and continues her life without symptoms. Our view is that as the number of publications available increases the durations associated with this survival in the literature will change.

\section{CONCLUSIONS}

The prognosis of cardiac LMS is generally poor, and the survival of primary cardiac LMS without treatment is approximately 6-12 months. Therefore, we planned to give cardiac radiotherapy and a subsequent adjuvant IMA treatment to our patient after the second recurrence surgery.

\section{DECLARATIONS}

\section{Competing of Interest}

The authors declare no competing interest in this study.

\section{Acknowledgment}

Not applicable

\section{REFERENCES}

1. Maleszewski JJ, Bois MC, Bois JP, et al. Neoplasia and the heart: A pathological review of effects with clinical and radiological correlation. J Am Coll Cardiol. 2018;72(2):202-27.

2. Kosuga T, Fukunaga $S$, Kawara $T$, et al. Surgery for primary cardiac tumors. Clinical experience and surgical results in 60 patients. J Cardiovasc Surg (Torino). 2002;43(5):581-7.

3. McAllister HA Jr. Cardiovascular pathology. Churchill Livingstone, New York, 1983.

4. Weir DR, Joncs BC Jr, Ohio C. Primary sarcoma of the heart. Am Heart J. 1940;22:556-60.

5. Poole GV Jr, Meredith JW, Breyer RH, Mills SA. Surgical implications in malignant cardiac disease. Ann Thorac Surg. 1983;36:484-91.

6. Pins MR, Ferrell MA, Madsen JC, et al. Epithelioid and spindle-celled leiomyosarcoma of the heart: Report of 2 cases and review of the literature. Arch Pathol Lab Med. 1999;123(9):782-8.

7. Antman K, Crowley J, Balcerzak SP, et al. An intergroup phase III randomized study of doxorubicin and dacarbazine with or without ifosfamide and mesna in advanced soft tissue and bone sarcomas. J Clin Oncol. 1993;11(7):1276-85.

8. Truong PT, Jones SO, Martens B, et al. Treatment and outcomes in adult patients with primary cardiac sarcoma: the British Columbia Cancer Agency experience. Ann Surg Oncol. 2009;16(12):3358-65. 